\title{
A Note on Translation and Terminology
}

Although the Pinyin phonetic system based on Putonghua or Mandarin Chinese has been more widely used in recent academic writings, the earlier Wade-Giles system still persists. In this book, I have used Pinyin whenever the Chinese names of places occur, except those preferred transliterations of certain proper nouns, for example, "Canton" for "Guangzhou" (Pinyin). Regarding the names of Chinese people mentioned in the book, however, the situation is more complicated. While the English-language government records, archival manuscripts, and newspapers recorded the names of Chinese people based on their pronunciation in Cantonese or other local dialects without consistency, the Chinese-language sources provided the names in Chinese characters that are consistent in writing. While such variants make consistency in spelling people's names nearly impossible, I have managed to arrive at some degree of consistency by using the original spellings cited in the English-language sources and Pinyin spellings based on Putonghua from the Chinese-language sources. Nevertheless, inconsistency is inevitable in some cases; for example, the surname "Moy" (according to its Cantonese pronunciation) also appears in its Pinyin form "Mei" in later chapters.

In addition, both the singular form "community" and plural form "communities" have been used in reference to the Chinese settlement in Chicago before and after I9I2, respectively. Before I9I2, it was primarily a single-sited community, located in the Loop area around South Clark Street. After I9I2, when the majority of the Chinatown businesses and residents relocated to 
xviii Note on Translation and Terminology

the Cermak-Wentworth area, some still remained in the South Clark neighborhood. Since the I96os, the expansion of South Chinatown, the emergence of suburban communities, and the revival of North Chinatown have resulted in multi-sited Chinese American communities. Moreover, the plural form, "communities," also emphasizes the cultural, economic, linguistic, political, and social diversity among the Chinese in Chicago. 
Chinese Chicago 
